\title{
Kosterlitz-Thouless transition on the worldsheet of the QCD string
}

\author{
Harvey B. Meyer* \\ DESY \\ Platanenallee 6 \\ D-15738 Zeuthen \\ E-mail: harvey.meyer@desy.de
}

\begin{abstract}
We investigate the properties of the QCD string in the Euclidean $\mathrm{SU}(N)$ pure gauge theory when the space-time dimensions transverse to it are periodic. We propose a generalisation of the Lüscher-Weisz effective string action for the flux-tube energy levels at finite $L_{\perp}$. As the size of one transverse dimension is varied, we predict a Kosterlitz-Thouless transition of the worldsheet field theory at $\sigma\left(L_{\perp}\right) L_{\perp}^{2} \simeq 1 / 8 \pi$ driven by vortices, after which the periodic component of the worldsheet displacement vector develops a mass gap and the effective central charge drops by one unit. The universal properties of the transition are emphasized.
\end{abstract}

XXIIIrd International Symposium on Lattice Field Theory

25-30 July 2005

Trinity College, Dublin, Ireland

${ }^{*}$ Speaker. 


\section{Introduction}

Our aim is to investigate the effect on the spectrum of the QCD string (for a review, see [1]) of periodic transverse dimensions ([2]; see [3] for earlier work on the subject). On the one hand it is important to know the magnitude of the finite-size effects on the string spectrum in lattice simulations. By the same token it is hoped that one might exploit these effects to learn more about the nature of the 'flux-tube' degrees of freedom.

Our starting point will be the effective theory proposed by Lüscher and Weisz [4] for the Polyakov loop correlator in $D=3$ and $4 \mathrm{SU}(N)$ pure gauge theories in infinite transverse dimensions.

\section{The Lüscher-Weisz effective theory for the QCD string}

This effective theory [4] is in principle capable of predicting the splittings (and the degeneracies) between low-lying energy levels of the flux-tube, once a few low-energy constants have been determined. So it is a low-energy effective theory with a finite UV-cutoff set by the string tension. Indeed, at energies of that order, once expects the internal degrees of freedom of the string to become excited.

The partition function is defined by an action living on the worldsheet $R \times L$ of the string. The connection with the gauge theory observables is made by equating the Polyakov loop correlation function $\left\langle P_{t}(0)^{*} P_{t}(x)\right\rangle$ with this partition function, where $|\vec{x}|=R$ and the length of the loops is $L$. For $L \gg R$ the correlator is given by $e^{-V(R) L}$, where $V(R)$ is interpreted as the potential between two static quarks. The partition function corresponds to a non-renormalisable Euclidean quantum field theory in (1+1) dimensions, on a spatial 'volume' of linear size $R$ and at temperature $T=1 / L$; or, alternatively, as a statistical mechanics system living on a two-space-dimensional lattice with a lattice spacing of order $\sigma^{-1 / 2}$ (which has nothing to do with the lattice on which the gauge theory may or may not be regulated).

The field living on the worldsheet $\left(0 \leq z_{0} \leq L, 0 \leq z_{1} \leq R\right)$ is a bosonic field $\vec{h}$ with $D-2$ components. Since it represents the displacement of the worldsheet from the classical configuration, it has engineering dimension of length. The Polyakov loops are the propagators of static quarks, so we are discussing the open-string case, and this is reflected in the effective theory by imposing Dirichlet boundary conditions on $\vec{h}$ at $z_{1}=0$ and $z_{1}=R$. The action of the effective theory is

$$
\begin{aligned}
S= & \sigma R L+S_{\text {fluc }} \\
S_{\text {fluc }}= & \frac{\sigma}{2} \int d^{2} z\left[\left(\partial_{\mu} \vec{h}\right)^{2}+c_{1}\left(\partial_{1} \vec{h}\right)^{2}(\delta(R)+\delta(0))\right. \\
& \left.+c_{2}^{(1)}\left(\partial_{\mu} \vec{h}\right)^{2}\left(\partial_{\nu} \vec{h}\right)^{2}+c_{2}^{(2)}\left(\partial_{\mu} \vec{h} \cdot \partial_{\nu} \vec{h}\right)^{2}+\ldots\right]
\end{aligned}
$$

The Gaussian action gives the leading contribution to the string spectrum at large $R$. Each higherorder term is multiplied by an unknown dimensionless constant $c_{k}^{(i)}$; some of them vanish/ are constrained by the open-closed string duality [4]. The lower index on the action terms above indicate how many more derivatives of $\vec{h}$ they contain than $S_{0}$. On general grounds the correction to $V(R)$ associated with an operator of dimension $d_{\text {op }}$ (when $\vec{h}$ is canonically normalised) is $\frac{1}{R}\left(1 / \sigma R^{2}\right)^{d_{\mathrm{op}} / 2-1}$. The parameter $\sigma$ in Eq. 2.2 controls the amplitude of the fluctuations around the 
classical solution; we assume that it is the same quantity as the one appearing in Eq. 2.1, which determines the classical energy. The fact that we are to treat it as a large quantity (it is the UV cutoff) clearly shows that we are doing a semi-classical calculation.

Lattice simulations [7, 9] have confirmed the leading, universal correction [5] to the static potential predicted by the effective theory $(2.1,2.2)$ :

$$
V(R)=\sigma R-\frac{\pi d_{\perp}}{24 R}+O\left(\frac{1}{\sigma R^{3}}\right)
$$

where $d_{\perp}$ is the number of (infinite) transverse space-time dimensions. We now ask ourselves, what happens if the latter are finite and periodic?

\section{Finite transverse dimensions to the string}

The first observation is that $V\left(R, L_{\perp}\right)$ is linearly confining $\forall L_{\perp}=\left(L_{1}, L_{2}\right)$. The qualitative behaviour of the string tension is known in a few limits: $\sigma\left(L_{1}, L_{2}\right) \propto 1 /\left(L_{1} L_{2}\right)$ when $L_{1}, L_{2} \rightarrow 0$; $\sigma\left(L_{1}\right) \propto 1 / L_{1}^{2}$ when $L_{1} \rightarrow 0$; and $\sigma\left(L_{1}\right)=\sigma(\infty)+O\left(e^{-m_{G} L_{1}}\right)$ when $L_{1}$ is large, where $m_{G}$ is the lightest glueball mass. Secondly, $\sigma=\sigma\left(L_{\perp}\right)$ and $c_{k}=c_{k}\left(L_{\perp}\right)$ become 'arbitrary' functions of $L_{\perp}$ (they can be computed on the lattice). Since the effective theory cannot predict the values of these parameters, we focus on the static potential in reduced units:

$$
v(r) \equiv V(R) / \sqrt{\sigma}=r-\frac{\pi d_{\perp}}{24 r}+\ldots, \quad r \equiv \sqrt{\sigma} R .
$$

The question that now arises is, how does the Lüscher correction vary with $L_{\perp}$ ? When a transverse dimension shrinks to zero, the $D$-dimensional gauge theory should dimensionally reduce to a $(D-1)$-dimensional one, where the Lüscher correction is given by $-\pi\left(d_{\perp}-1\right) / 24 r$. From the point of view of the effective string theory, the coefficient is nothing but the central charge of the $2 \mathrm{~d}$-conformal theory describing the fluctuations of the worldsheet; since it is given by the number of massless bosonic degrees of freedom living on the worldsheet, it cannot vary continuously. We thus expect the Lüscher correction to have a discontinuity at a phase transition of the worldsheet quantum field theory.

Consider for simplicity the case of $3 \mathrm{~d} \mathrm{SU}(N)$ gauge theory, with the size of the (unique) transverse dimension given by $L_{\perp}$. A possible interpretation is that $L_{\perp}=1 / T$ and we are looking at the screening mass of a heavy quark at finite temperature. What is now the effective string theory?

Due to the geometry of the target space, the fluctuation field is parametrised by an angular variable:

$$
h(z)=\frac{L_{\perp} \theta(z)}{2 \pi} \Rightarrow S_{0}=\frac{K}{2} \int d^{2} z\left(\partial_{\mu} \theta\right)^{2}, \quad K=\frac{\sigma\left(L_{\perp}\right) L_{\perp}^{2}}{(2 \pi)^{2}} .
$$

A low-energy effective theory with UV cutoff $\sqrt{\sigma}$ is equivalent to a lattice field theory with lattice spacing $O\left(\sigma^{-1 / 2}\right)$ [6]. Two-dimensional systems with compact dynamical variables defined on a lattice are well-known in statistical mechanics. The most famous instance is the XY-model:

$$
S_{\mathrm{xy}}=K_{\mathrm{xy}} \sum_{x, \mu}[1-\cos (\theta(x)-\theta(x+a \hat{\mu}))]
$$

However, any model with the same symmetries is just as acceptable, as long as we focus on the universal properties of this class of models. Next, we review the properties of the XY-model that are relevant to the dynamics of the QCD string. 


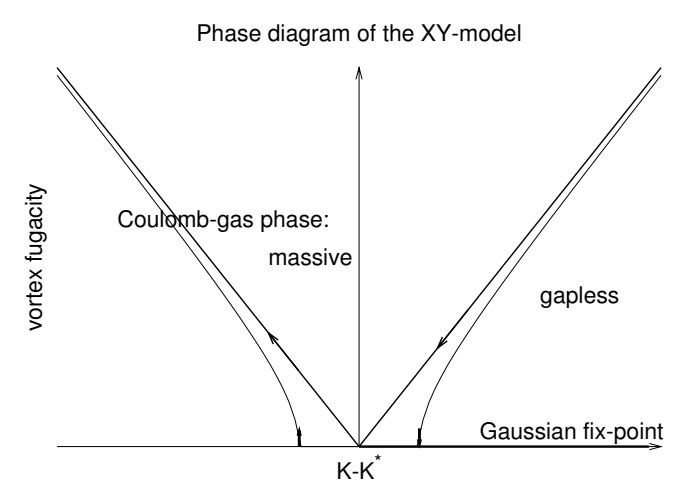

Figure 1: The phase diagram of the XY model.

\section{The XY-model as an effective description}

At large $K_{\mathrm{xy}}$, the partition function is dominated by Gaussian fluctuations around the trivial $\theta=0$ vacuum. However, the system admits vortex-antivortex configurations

$$
e^{i \theta(z)}=e^{i \theta_{\infty}} \frac{z-z^{+}}{\left|z-z^{+}\right|} \frac{\left|z-z^{-}\right|}{z-z^{-}}
$$

whose energy is $\sim \log (R / a)$, where $R=\left|z^{+}-z^{-}\right|$. At low temperatures, such configurations with $R$ of physical size are suppressed; however their entropy is also proportional to $\sim \log (R / a)$, so that they drive a phase transition (of infinite order) at some finite $K_{\mathrm{xy}}^{*} \simeq 2 / \pi$, as shown by Kosterlitz and Thouless [8]. Clearly they have a strong disordering effect on the field $\theta$ : a screening mass is generated at small $K_{\mathrm{xy}}$.

Let us apply these known facts to the effective theory on the string worldsheet. In doing so, it is helpful to have in mind the following physical interpretation of vortex configurations: the action (3.2) is a saddle-point expansion around the classical string solution which connects a quark to an antiquark separated by distance $R$. Clearly, there are also classical solutions with a net winding number around the compact transverse dimension. They have energy

$$
E_{\mathrm{cl}}(n)=\sigma\left(L_{\perp}\right) \sqrt{R^{2}+n^{2} L_{\perp}^{2}} \simeq E_{\mathrm{cl}}(n=0)+\frac{\sigma\left(L_{\perp}\right) L_{\perp}^{2} n^{2}}{2 R}, \quad R \gg L_{\perp} .
$$

The different classical configurations can only be connected if the string goes through more energetic configurations. Therefore such transitions are classically forbidden tunnelling processes. Quantum mechanically, one expects the tunnelling to become a frequent fluctuation when the gap $E(1)-E(0)$ is of same order as the Casimir energy of the $n=0$ vacuum. In this context, the worldsheet direction of size $R$ is interpreted as the space-direction, and that of size $L$ as the Euclidean time direction in a path integral treatment of the quantum-mechanical string. A vortex being a point-like object on the worldsheet, it corresponds to a process of the string describing a transition from a state at $t=-\infty$ to another state at $t=+\infty$. As long as we are considering the asymptotic limit $L \rightarrow \infty$, only vortex-antivortex configurations have a finite free energy in the $2 \mathrm{~d}$ QFT. It is easy to see from Eq. 4.1 that a worldsheet containing a $v \bar{v}$ pair describes the 'life' of a string that had winding number $n=0$ at $t=-\infty$, goes through a state with winding number \pm 1 , and returns to $n=0$ classical state (see Fig. 2). 

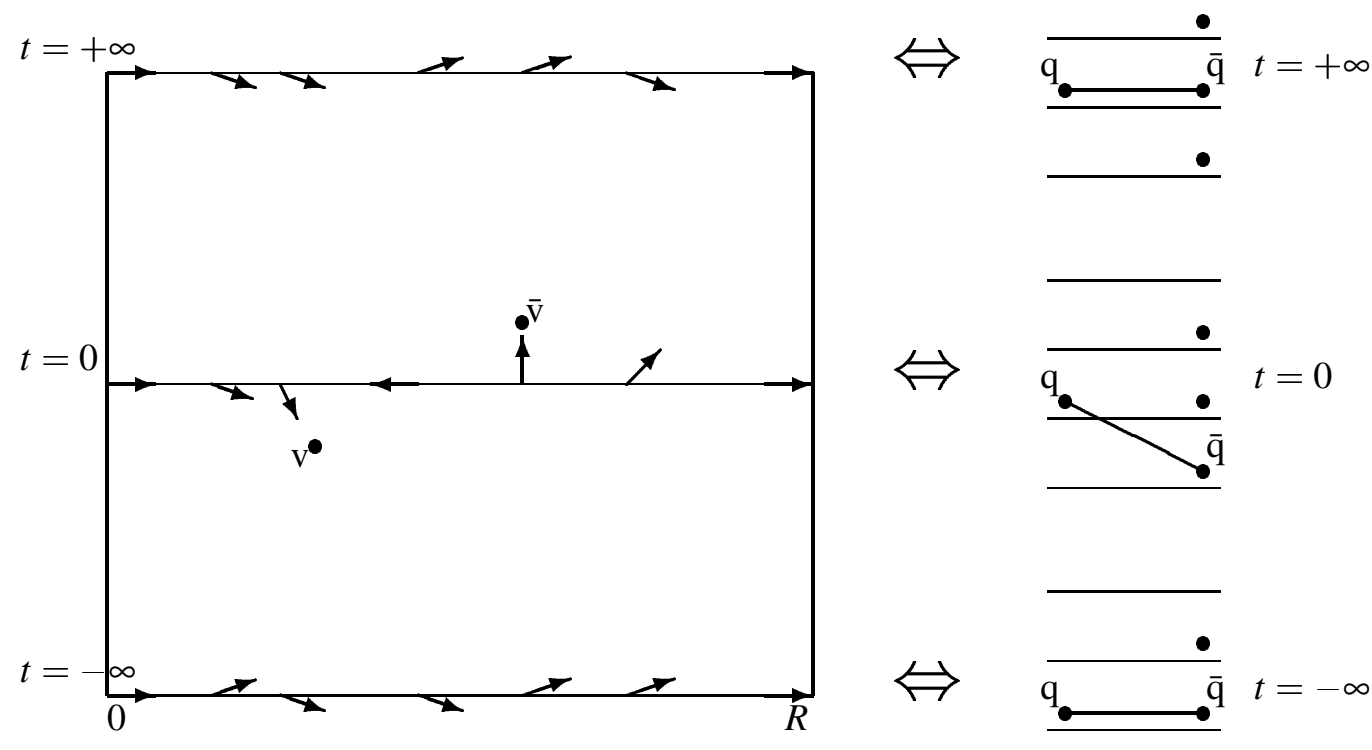

Figure 2: Physical interpretation of the vortices: the left-hand side, where $\theta$ is represented as a unit vector $(\cos \theta, \sin \theta)$, illustrates that the appearance of a vortex at time $t$ 'followed' by an antivortex at time $\bar{t}$ induces one unit of clockwise winding of the variable $\theta\left(t_{s}, x\right)$ as $x$ varies from 0 to $R$ on a timeslice at time $t_{s}$ located between $t$ and $\bar{t}$. Right, the corresponding interpretation for the string on the covering space of the torus: the string has non-trivial winding number along the transverse dimension in the interval $[t, \bar{t}]$ and trivial winding number elsewhere.

Qualitatively, we can predict the following scenario for $v(r)$ as $L_{\perp}$ is varied [2]: at large $L_{\perp}$, the periodicity plays no role for the long-distance properties of the string fluctuations; the Casimir energy still is $-\frac{\pi}{24 R}$. As $L_{\perp}$ decreases, vortices affect the non-universal contributions to the energy levels. At $L_{\perp}^{*} \simeq \sqrt{\frac{8 \pi}{\sigma\left(L_{\perp}^{*}\right)}}$ (the precise value is non-universal), the KT phase transition takes place on the worldsheet. For $L_{\perp}<L_{\perp}^{*}$, the fluctuations acquire a mass gap and the Casimir energy is of order $e^{-2 m R}$.

We can be more quantitative. Since vortices have a scaling dimension $x_{v}=\pi \kappa$ in the Gaussian approximation $S_{0}=\frac{\kappa}{2} \int d^{2} z\left(\partial_{\mu} \theta\right)^{2}$, their contribution (in pairs) to energy levels at $L_{\perp}>L_{\perp}^{*}$ is

$$
\Delta E\left(L_{\perp}, R\right)=\frac{c}{R}\left(\sigma\left(L_{\perp}\right) R^{2}\right)^{1-\pi \kappa} .
$$

with $\kappa=\frac{\sigma\left(L_{\perp}\right) L_{\perp}^{2}}{(2 \pi)^{2}}+O(1)$.

If we approach the phase transition from the $L_{\perp}<L_{\perp}^{*}$ side, the correlation length diverges faster than any power law [8]:

$$
m \sim m_{0} e^{-b \sqrt{K^{*} /\left(K^{*}-K\right)}}, \quad \text { Casimir energy } \sim e^{-2 m R} .
$$

By the same token, for a worldsheet of large but finite size, the phase transition becomes a crossover of spread $\delta K / K^{*}=b^{2} / \log ^{2} m_{0} R$.

We have discussed the dynamics of a single transverse dimension, since we were dealing with a three-dimensional gauge theory. If we now move to the four-dimensional case, where both 
components of the worldsheet fluctuation field $\vec{h}$ are periodic, the key point to note is that the two components are decoupled in the quadratic, renormalisable part of the action (2.2). Therefore the universal contributions to the string energy levels just add up. Also the existence of topological configurations in one component is unaffected by the presence of the other component.

\section{Conclusion}

We have studied an effective string theory for the flux-tube in $\mathrm{SU}(N)$ gauge theories in three and four compact dimensions. Let us summarise the outcome of our investigation of the Polyakov loop correlator when one transverse dimension is periodic and of size $L_{\perp}$. The periodic component of the fluctuation field is described by an action of the XY-model type. It follows that

- there is a phase transition of the worldsheet field theory at $L_{\perp}^{*}=O\left(\sigma^{-1 / 2}\right)$ where the periodic component acquires a mass gap and hence the central charge drops by one unit;

- it is a Kosterlitz-Thouless phase transition: approaching $L_{\perp}^{*}$ from below, the mass gap $m\left(L_{\perp}\right)$ goes to zero in a universal way in terms of $K \equiv \sigma\left(L_{\perp}\right) L_{\perp}^{2} / 4 \pi^{2}$ as given by Eq. 4.4. Once $\sigma\left(L_{\perp}\right)$ is independently determined, we have a prediction for the functional form of $m\left(L_{\perp}\right)$.

- in the large $L_{\perp}$ phase, there are (strongly suppressed) corrections to the string energy levels associated with the periodicity; they come in powers of $1 / R$ which vary continuously and increase monotonously with $L_{\perp}$; these corrections are exactly of order $1 / R^{3}$ at $L_{\perp}=L_{\perp}^{*}$.

The gauge theory of course has the 'deconfining' phase transition at $L_{\perp} \doteq 1 / T_{c}=O\left(\sigma^{-1 / 2}\right)$, and it would be intriguing if $L_{\perp}^{*}$ actually coincided with $1 / T_{c}$. This can be tested in lattice simulations. Numerical evidence was already presented in [3] showing that in the three-dimensional $Z(2)$ gauge model $L_{\perp}^{*}$ lies within $10 \%$ of $1 / T_{c}$.

\section{References}

[1] J. Kuti, Plenary talk at this conference.

[2] H. B. Meyer, arXiv:hep-th/0506034.

[3] M. Caselle, F. Gliozzi and S. Vinti, Nucl. Phys. Proc. Suppl. 34 (1994) 263 [arXiv:hep-lat/9403022]; M. Caselle, R. Fiore, F. Gliozzi, P. Guaita and S. Vinti, Nucl. Phys. B 422 (1994) 397 [arXiv:hep-lat/9312056].

[4] M. Luscher and P. Weisz, JHEP 0407 (2004) 014 [arXiv:hep-th/0406205].

[5] M. Luscher, K. Symanzik and P. Weisz, Nucl. Phys. B 173 (1980) 365; M. Luscher, Nucl. Phys. B 180 (1981) 317.

[6] K. Symanzik, Nucl. Phys. B 226 (1983) 187.

[7] M. Luscher and P. Weisz, JHEP 0207 (2002) 049 [arXiv:hep-lat/0207003]; K. J. Juge, J. Kuti and C. Morningstar, arXiv:hep-lat/0401032; Nucl. Phys. Proc. Suppl. 129 (2004) 686 [arXiv:hep-lat/0310039]; M. Caselle, M. Hasenbusch and M. Panero, JHEP 0503 (2005) 026 [arXiv:hep-lat/0501027].

[8] J. M. Kosterlitz and D. J. Thouless, J. Phys. CC 6, 1181 (1973).

[9] H. B. Meyer and M. Teper, JHEP 0412 (2004) 031 [arXiv:hep-lat/0411039]. 